\title{
Motor cortex changes in spinal cord injury: a TMS study
}

\author{
Eleonora Saturno* $^{\dagger}$, Claudio Bonato*, Carlo Miniussi ${ }^{\star}$, VincenzoDi Lazzaro $^{\dagger}$ \\ and Leonardo Callea*
}

\author{
*Fondazione "Don Carlo Gnocchi", Rovato (Brescia), Italy \\ $\dagger$ Institute of Neurology, Policlinico "A. Gemelli", Roma, Italy \\ *IRCCS S Giovanni di Dio, Fatebenefratelli, Brescia, Italy
}

\begin{abstract}
Using paired pulse transcranial magnetic stimulation (TMS) paradigms, we studied cortical excitability in a patient with spinal cord lesion. During posterior tibial nerve stimulation, the contextual flexion of hand fingers contralateral to the stimulated lower limb had suggested a change in motor cortex excitability. Results showed a decrease in the activity of motor cortex inhibitory circuits. This could suggest that in spinal cord injury, just as in stroke and peripheral deafferentation, a disinhibition of latent synapses within the motor cortex and the rewriting of a new motor map can occur. [Neurol Res 2008; 000: 000-000]
\end{abstract}

Keywords: Motor cortex plasticity; spinal cord lesion; TMS

\section{INTRODUCTION}

The motor cortex reacts to spinal cord injuries undergoing modifications, including motor map reorganization.

Experimental observations support the hypothesis that an imbalance between inhibitory and excitatory circuits, resulting in the unmasking of latent corticocortical connections, is important in cortical plasticity mechanisms ${ }^{1,2}$.

To our knowledge, there are not at present any demonstrations, through transcranial magnetic stimulation (TMS) investigation, of a reduction in inhibitory activity of motor cortex after spinal cord injury, although this has been well documented in stroke ${ }^{3,4}$ and peripheral deafferentation ${ }^{5}$.

Here we examined, through TMS paradigms, the cortical inhibitory circuits after a spinal cord injury.

We describe the case of a 67-year-old woman who, at the age of 44 years, suffered an episode of ischemic myelopathy. The earliest symptoms consisted of dorsal pain and distal weakness in the left upper limb, followed by weakness and paresthesia in both lower limbs, and finally distal weakness in the right upper limb. The patient underwent rehabilitation, regaining ambulation.

At the time of our observation, she was healthy and was not taking any drugs. Examination showed mild spastic paraparesis (Ashworth scale $=1$ ), bilateral hypotrophy and weakness in C8-D1 muscles, mild hypoesthesia with a level D4. Tendon reflexes were

Correspondence and reprint requests to: Eleonora Saturno, Neurorehabilitation Unit, Fondazione "Don Carlo Gnocchi", Via Golgi, 1-25038 Rovato, Brescia, Italy. [eleonorasaturno@gmail.com] Accepted for publication March 2008. increased for legs, normal/reduced for upper limbs, and there was a bilateral Babinski sign.

Brain MRI was normal. Spine MRI showed slight hyperintensity of anterior horns at dorsal level in $\mathrm{T}_{2}$ weighted images. Somatosensory-evoked potentials were normal.

Central motor conduction time indicated damage to central pathways of the lower limbs and radicular damage to the right upper limb (Kimura method).

EMG documented bilateral signs of mild motor unit rearrangement at C8-T1 level. Nerve conduction studies of lower limbs, including $\mathrm{F}$ wave, were normal.

During SEP's registration, we noted a contextual flexion of left hand fingers when stimulating the right lower limb, and of right hand fingers when stimulating the left lower limb. The phenomenon also occurred on evocation of the Babinski sign. Usually, the noxious stimulation of the sole causes a motor response only in the stimulated limb, through a reflex arch modulated by inhibitory cortical pathways. Thus, the consensual movement of the contralateral hand in the patient led us to wonder whether a change in cortex excitability could have occurred.

\section{MATERIALS AND METHODS}

We explored motor cortex excitability by evaluating short latency intracortical inhibition $(\mathrm{SICl})$ and resting motor threshold (RMT) to TMS. Both parameters are thought to be related to the inhibitory activity in the motor $\operatorname{cortex}^{6-9}$. We also evaluated short latency intracortical facilitation (ICF).

TMS was performed with a high power Bistim module (Magstim Co., Whitland, Dyfed, UK). A figure-of-eight coil with external loop diameters of $9 \mathrm{~cm}$ was held over the right motor cortex at the optimum scalp position to 


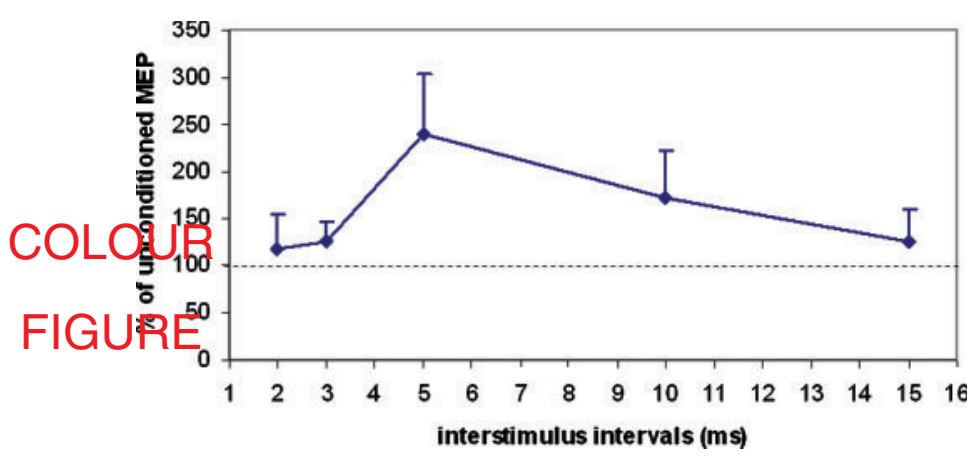

Figure 1: $\mathrm{SICl}$ and SICF in the reported patient. Horizontal line $(100 \%)$ indicates the size of the unconditioned MEP; error bar= 1 SE. Note the absence of any inhibition at early ISIs of 2, 3 and $5 \mathrm{~ms}$

elicit EMG responses in the left extensor digitorum communis (EDC). The induced current flowed in a postero-anterior direction. Surface muscle responses were obtained via two $9 \mathrm{~mm}$ diameter $\mathrm{Ag}-\mathrm{AgCl}$ electrodes with the active electrode over the motor point of the target EDC muscle and the reference electrode was positioned $2 \mathrm{~cm}$ ahead. EMG responses were amplified and filtered (bandwidth: $50 \mathrm{~Hz}-2 \mathrm{kHz}$ ) by BrainAmp 32MR amplifiers (BrainProducts $\mathrm{GmbH}$, Munich, Germany). Data were collected on a computer with a sampling rate of $2.5 \mathrm{kHz}$ and stored for offline analysis.

RMT is the minimum stimulus intensity that produced a liminal EMG response ( $\sim 50 \mu \mathrm{V}$ in $50 \%$ of ten trials) at rest.

SICl and ICF were studied using paired pulse paradigms ${ }^{10}$. Two magnetic stimuli were given through the same coil over the motor cortex. The effect of the first (conditioning) stimulus on the second (test) stimulus was investigated. The intensity was set at 80 and $120 \%$ RMT for the conditioning and the test stimuli, respectively. The timing of the conditioning shock was altered in relation to the test shock. Interstimulus intervals (ISIs) of 2, 3 and $5 \mathrm{~ms}$ were used for establishing SICI. ISIs of 10 and $15 \mathrm{~ms}$ were used for ICF. Five stimuli were delivered for each ISI at random.

The amplitude of conditioned response was expressed as percentage of control response.

The study was conducted according to the Declaration of Helsinki.

\section{RESULTS}

The amplitude of conditioned MEPs for different ISIs is reported in Figure 1. The data showed a marked reduction of intracortical inhibition for all ISIs. No inhibition of conditioned MEPs was observed at very short (2, 3 and $5 \mathrm{~ms})$ ISIs. Instead, a modest, although not significant increase was present at ISIs 2 and $3 \mathrm{~ms}$ $(p>0.05)$ and become highly potentiated at ISI $5 \mathrm{~ms}$ $(p<0.01)$. At longer ISIs $(10$ and $15 \mathrm{~ms})$, conditioned MEP amplitude still remained greater than unconditioned MEP, being significant only at ISI $10 \mathrm{~ms}(p<0.05)$.
RMT value was $23 \%$ of the maximum output of the stimulator, which was significantly lower than the normal range for the patient's age (n.v. \pm SD: $46.6 \pm$ $6.5 \%)$.

\section{DISCUSSION}

The observed contextual movements of the contralateral hand observed during peripheral stimulation of lower limbs suggest a less specialized recruitment of cortical motor neurons for output.

Impairment of $\mathrm{SICl}$ and low RMT are consistent with a reduction in the inhibitory tonic activity of the motor cortex.

The imbalance between excitatory and inhibitory circuits could eventually result in a disinhibition of latent synapses and the rewriting of the motor map.

These data provide a further suggestion, the first through TMS investigation, of a motor cortex rearrangement after spinal cord lesion, which is not dependent on peripheral deafferentation (SEP and nerve conduction being normal), and support the hypothesis that inhibitory pathways play a pivotal role in cortex plasticity.

This single-case study does not claim to be a definitive demonstration in support of our hypothesis: this profile (low RMT and lack of SICl) might be an intrinsic characteristic of this patient, and further observations are needed to confirm this preliminary one. But since a similar combination of clinical and neurophysiologic characteristics is uncommon, this case is sufficiently important to report.

\section{REFERENCES}

1 Levy LM, Ziemann U, Chen R, et al. Rapid modulation of GABA in sensorimotor cortex induced by acute deafferentation. Ann Neurol 2002; 52: 755-761

2 Jacobs KM, Donoghue JP. Reshaping the cortical motor map by unmasking latent intracortical connections. Science 2001; 251: 944-947

3 Liepert J, Hamzei F, Weiller C. Motor cortex disinhibition of the unaffected hemisphere after acute stroke. Muscle Nerve 2000; 23: 1761-1763

4 Shimizu T, Hosaki A, Hino T, et al. Motor cortical disinhibition in the unaffected hemisphere after unilateral cortical stroke. Brain 2002; 125: 1896-1907

5 Ziemann U, Muellerbacher W, Hallet M, et al. Modulation of practice dependent plasticity in human motor cortex. Brain 2001; 124: $1171-1181$

6 Ziemann U, Lonnecker S, Steinhof BJ, et al. The effect of lorazepam on the motor cortical excitability in man. Exp Brain Res 1996; 109: 127-135

7 Ziemann U, Lonnecker S, Steinhoff BJ, et al. Effects of antiepileptics drugs on motor cortex excitability in humans: A transcranial magnetic stimulation study. Ann Neurol 1996; 40: 367-378

8 Di Lazzaro V, Oliviero A, Meglio M, et al. Direct demonstration of the effect of lorazepam on the excitability of the human motor cortex. Clin Neurophysiol 2000; 111: 794-799

9 Ilic TV, Meintzschel F, Cleff U, et al. Short-interval paired-pulse inhibition and facilitation of human motor cortex: The dimension of stimulus intensity. J Physiol 2002; 545: 153-167

10 Kujirai T, Caramia MD, Rothwell JC, et al. Corticocortical inhibition in human motor cortex. J Physiol 1993; 471: 501-519 